plasma cortisol is abolished in Cushing's syndrome. If in a suspected case the morning plasma cortisol is found to be within the upper range of normal the test has been of little value; but a finding of the same level around midnight strongly supports the diagnosis. It may be difficult to ascertain whether a given case of Cushing's syndrome is due to bilateral adrenal hyperplasia from oversecretion of corticotrophin or to an adrenal tumour, benign or malignant, in which secretory function is autonomous. Suppression tests with large enough doses of dexamethasone will reduce the level of cortisol secretion in the former but not in the latter group. Again, in mild Addison's disease cortisol secretion can be within the normal basal range because the surviving remnant of adrenal cortex is working under maximal stimulation. The diagnosis can be confirmed by showing no increase of secretion in response to injections of long-acting corticotrophin.

The second half of the Lumleian lecture deals with aldosterone, with particular reference to its possible role in the pathogenesis of hypertension and oedema. The principal action of this hormone is to restrict sodium and increase potassium excretion, effects which can be measured in urine, sweat, and saliva. Aldosterone is formed in the zona glomerulosa of the adrenal cortex, the normal rate of secretion being about one-hundredth that of cortisol. This last factor, together with the great difficulty of measuring aldosterone in body fluids, has restricted studies in this field to a few research centres, and there is no early prospect of routine laboratory methods being developed as with cortisol. The stimuli for aldosterone secretion are loss of sodium and water from the body, as after heavy diuresis or haemorrhage, and excessive loading with potassium. Conversely, secretion is suppressed by a high intake of sodium, excessive water retention, and potassium depletion. Control seems to be independent of the pituitary and hypothalamus, and the mechanism is still not clear. There is, however, increasing evidence that angiotensin II may be the principal stimulating factor. This substance is formed by interaction between the enzyme renin and an alpha-2-globulin in plasma. Renin is secreted by the juxtaglomerular apparatus of the kidneys and is easier to measure in body fluids than angiotensin II.

In the initial stages of oedema formation aldosterone probably plays a minor and secondary role and oedema cannot be induced in healthy subjects by giving large amounts of the hormone. Other factors such as raised venous pressure or lowered plasma-protein osmotic pressure are more important. The role of aldosterone in the pathogenesis of hypertension is complex, for oversecretion may be primary, or secondary to the vascular disorder. In most cases of essential hypertension secretion of the hormone is not increased, but in primary aldosteronism (Conn's syndrome) it seems to be the primary factor, causing hypertension through its effects on electrolyte balance. Oedema is notably absent in this condition and secretion of renin is suppressed. However, in malignant hypertension due to renal artery stenosis there is usually an increase in secretion both of aldosterone and of renin, though the excessive aldosterone production is probably secondary to the raised level of renin. Aldosterone may then potentiate this form of hypertension but does not initiate it. Cope discusses the difficulties that can arise in distinguishing between primary and secondary aldosteronism. The problem has become complicated even further by reports of adrenal adenomas occurring in a few cases of renal artery stenosis. These may arise in response to prolonged adrenal stimulation by angiotensin II, in turn secondary to renal ischaemia. Such adenomas may eventually become autonomous. It is therefore important that the adrenal glands should be inspected during operations for renal artery stenosis.

\section{Cough and Cancer}

The Committee on Smoking and Health of the Royal College of Physicians pointed out in its report $^{1}$ that universal abstinence from smoking should not be necessary to prevent lung cancer if only the minority of susceptible subjects could be detected and persuaded not to smoke. The committee cited preliminary evidence that a smoker's cough might be a danger signal.

Three subsequent studies have added some weight to this suggestion. E. C. Hammond ${ }^{2}$ in a postal survey of physical complaints among nearly half a million American men found that over the subsequent two years more of those with cough suffered from lung cancer than of those who did not cough, the excess increasing with severity of the cough. In 1951 K. R. Boucot and her colleagues started a periodic radiological and symptomatic survey of 6,137 men in Philadelphia. In their latest report ${ }^{3}$ they show that cigarette smokers who reported having a cough at the beginning of the study had an attack rate of lung cancer over the subsequent ten years that was twice that of non-coughing smokers. And the difference in the incidence of cancer between coughers and noncoughers was greater for moderate than for heavy smokers. In a retrospective study of men and women dying of lung cancer in Northern Ireland compared with a control group of men dying from non-respiratory causes G. Dean ${ }^{4}$ calculated that the lung cancer mortality of smokers who were reported to have a morning cough three years before death was between three and five times greater than that of non-coughers. $\mathrm{He}$ also found the difference was greater in lighter than in moderate or heavy smokers.

In all these reports it remains uncertain whether the increased mortality from lung cancer of the coughers was because some of them already had early cancer or because some had premalignant bronchial metaplasia, such as that described by $O$. Auerbach and his colleagues, ${ }^{5}$ or the mucoid secretion which R. D. Passey ${ }^{6}$ believes to be the condition responsible for many lung cancers. The remarkably low incidence of bronchial metaplasia in ex-smokers ${ }^{5}$ and the rapid decline of lung cancer mortality observed by Richard Doll and Sir Austin Bradford Hill ${ }^{7}$ among cigarette smokers who stop smoking shows that precancerous changes may be reversible or are at least not inevitably progressive. But once malignant changes have started it may be too late to benefit from stopping smoking. Nor can cough be regarded as helpful in obtaining early diagnosis, for this can seldom be firmly established, even if suspected, before fatal metastases have occurred. Before a smoker's cough could be a useful

\footnotetext{
'Royal College of Physicians, Smoking and Health, 1962. Pitman Medical, London.

2 Hammond, E. C., Amer. F. publ. Hlth, 1964, 54, 11. Boucot, K. R., Cooper, D. A., Weiss, W., and Carnahan, W. J., 7.

4 Dean, G., Brit. med. F., 1966, 1, 1506.

Auerbach, O., Stout, A. P., Hammond, E. C., and Garfinkel, L., New Engl. F. Med., 1961, 265, 253.

- Passey, R. D., Lancet, 1962, 2, 107.

7 Doll, R., and Hill, A. B., Brit. med. F., 1964, 1, 1460.

Spratt, J. S., Spiut, H. J., and Roper, C. L., Cancer (Philad.), 1963, 16,687

10 Garland, L. H., Coulson, W., and Wollin, E., ibid., 1963, 16, 694.

10 Fletcher, C. M., Proc. roy. Soc. Med., 1965, 58, 918.
} 
warning sign of precancer, its prognostic value would have to be established over a longer period that that covered by the studies hitherto reported, for there is evidence that squamous and undifferentiated cancers may originate 10 years or more before death. ${ }^{8} 9$

Even if the early warning value of a cough were established it could help to prevent but a small proportion of cancers. Only half the cancers in the study of Boucot and colleagues occurred in coughers and only half of these may still have been premalignant when the cough began. Experience in antismoking clinics, attended by men who are specially keen to stop smoking, shows an average success rate of about $30 \%{ }^{10}$ Combining these percentages suggests that less than $10 \%$ of lung cancer could at present be prevented by trying to get coughing smokers to stop smoking. But this would not be the only benefit. A smoker's cough is an almost invariable preliminary to disabling bronchitis. If smokers who cough could be persuaded to stop smoking, disability and mortality from chronic bronchitis would certainly be diminished.

The practical problem of persuading people to stop remains the chief obstacle. We need more research into tobacco habituation to discover how more people may be freed from it. Meanwhile doctors should urge all their patients who have a cough to stop smoking forthwith. Only too often they are told to "cut down," and this is practically never possible.

\section{Safety of Halothane}

In most British hospitals halothane is now much the commonest vapour added to the gaseous anaesthetic mixture. But for some years a doubt has attended its administration. Is there a causal connexion between the drug and the post-operative jaundice and fatal hepatic necrosis that have occasionally followed anaesthesia with it?

The sporadic nature of the cases, the non-specific histological appearances of the liver, the presence of other possible causes, and the absence of clear animal experimental evidence of the hepato-toxicity of halothane in the circumstances of anaesthesia have kept this problem open. An obvious method of settling the answer is a prospective study. Apart from its magnitude such an investigation would not be too difficult to organize. However, opinions already expressed about the toxicity of halothane could present anaesthetists with an ethical dilemma, and plans for one large-scale prospective study in the U.S.A. were abandoned on these grounds. Anxieties were considerably allayed by the detailed and comprehensive retrospective studies by W. W. Mushin and colleagues $^{1}$ and E. M. Slater and colleagues ${ }^{2}$ in 1964 . These reports concluded that halothane was no more toxic to the liver than other anaesthetics. A further and very large retrospective study, called the National Halothane Study, was mounted in the United States, with results being analysed from 34 hospitals over a period of four years, and a summary of the report has recently been published. ${ }^{3}$

The important facts exposed by this study are the hospital mortality within six weeks after operation and the incidence

\footnotetext{
1 Mushin, W. W., Rosen, M., Bowen, D. J., and Campbell, H., Brit. med. F., 1964, 2, 329.

slater, E. M., Gibson, J. M., Dykes, M. H. M., and Walzer, S. G., New Engl. F. Med., 1964, 270, 983.

s Summary of the National Halothane Study, f. Amer. med. Ass., 1966, 197,775 .
}

of fatal hepatic necrosis. The study covered 856,000 patients, of whom 82 developed liver necrosis, or about 1 per 10,000 patients. Histological sections and clinical records were examined and a diagnosis made without knowledge of the details of the anaesthetic administered. The total death rate within six weeks of operation was found to be under $2 \%$, but the death rate after administration of halothane was lower than this figure, and was midway among the rates for the several general anaesthetics used. The rate of fatal hepatic necrosis after halothane was lower than that after cyclopropane. The study by Mushin and colleagues ${ }^{1}$ did not show any increased liver damage when halothane was administered to patients with disease of the biliary tract. The present American study reports the same finding. Hepatic necrosis occurred after about 1 in 5,000 cases of cholecystectomy. However, in only one out of the six of these cases did it follow halothane anaesthesia, in spite of the fact that halothane was administered to one-third of the patients having biliary-tract operations.

There were in the American survey a few cases of hepatic necrosis-9 out of the 82-which could not be explained in any way other than by the nature of the anaesthetic. Seven of these patients had received halothane, and five of them had had one or more previous operations within the preceding six weeks. It seems, therefore, that if there is a connexion between halothane and liver damage-and the matter is still open-it is rare, but it might have some connexion with repeated administration of the anaesthetic.

The results of the American study, therefore, support in broad outline those obtained by Mushin and colleagues. It does not establish any causal relationship between halothane and hepatic necrosis, though it does not completely exclude the possibility. It gives the reasonable advice that, until the matter is finally settled, unexplained fever and jaundice in a patient after administration of halothane should contraindicate the subsequent use of the anaesthetic for that patient. Perhaps the most valuable outcome of this impressively large survey is a recommendation which is not directly concerned with halothane-namely, that mortality after surgery and anaesthesia should be looked at again, particularly with regard to any differences between institutions as well as between the various anaesthetics.

\section{Walking Through Angina}

The pain of ischaemic heart disease is one of the most common and most varied of visceral pains. It is usually precipitated by muscular exercise, but some patients get pain only after taking a heavy meal or exposure to cold, and a few others have attacks brought on by smoking. Once the pain has begun during exercise most sufferers find that it persists as long as they continue, and either the pain itself or fear of the consequences of carrying on often causes them to halt.

A few patients find that their pain wears off if they continue to exercise. The earliest known reference to this is in a letter read by William Heberden ${ }^{1}$ at the College of Physicians in London on 17 November 1772. Heberden had just published his account of angina pectoris in the second volume of the Medical Transactions of the College and had received

\footnotetext{
1 Heberden, W., Medical Transactions of the College of Physicians, Vol. 3, p. 1785. London.

${ }^{2}$ MacAlpin, R. N., and Kattus, A. A., Circulation, 1966, 33, 183.
} 